que l'augmentation paradoxale de l'indice de réfraction du sérum chlorocalcique des laits aigris est due principalement à la formation de lactate de chaux aux dépens de la caséine. Le lactate de chaux reste en solution dans le sérum, en élève la réfraction, jusqu'à ce que toute la chaux de la caséine soit saturée. Ce point atteint, la réfraction baisse, reprend la saleur qu'elle asait primitivement pour le lait frais, puis diminue encore.

\title{
NOTE \\ SUR LE DOSAGE DE LA MATIÈRE GRASSE DANS LES POUDRES DE LAIT
}

\author{
par Albert SCHOONJANS \\ Docteur en sciences naturelles, Chimiste expart près les Tribunaux, Bruxelles
}

Les chimistes qui ne sont pas spécialistes en matière de lait et de beurre continuent à appliquer aux poudres de lait le procédé de Gerber ordinaire pour le dosage de la matière grasse.

Il a été démontré, il y a de longues années, que cette méthode fournit des chiffres trop bas. Elle a été remplacée par le procédé Gottlieb, modifié par Röse, lequel consiste, en substance, en ceci :

Méthode Gottlieb-Röse (1). - Dans un tube gradué de construction spéciale, on introduit successivement $1 \mathrm{gr}$. de poudre de lait et 9 cc. d'eau chaude, puis, après avoir agité vivement, 2 cc. d'une solution d'ammoniaque ( 6 fois normale), 10 ce. d'alcool, 25 cc. d'éther sulfurique et $23 \mathrm{cc}$. d'éther de pétrole. Au bout d'une heure de repos, lorsque le liquide s'est complètement éclairci en ce sens que la solution aqueuse sous-jacente ne présente tout au plus qu'une faible opalescence, sans aucun dépôt floconneux, on mesure le volume de la solution éthérée surnageante dans le tube, ce qui se fait par simple lecture et soustraction des deux niveaux. On note ce volume, on en prélève à la pipette $30 \mathrm{cc}$. que l'on fait évaporer dans une petite capsule exactement pesée au préalable. Le résidu est desséché à l'étuve à $100^{\circ} \mathrm{C}$. jusqu'à poids constant, puis pesé.

Le poids de la graisse ainsi trouvé est exprimé en décigrammes et rapporté à la totalité du volume d'éther. On obtient ainsi le pourcentage.

C'est cette méthode Gottlicb-Röse, que nous avons connu dès sa publication, que nous avons toujours appliquée jusqu'en ces derniers temps.

Au mois de décembre de l'année dernière, des divergences entre

(1) Zeitschrift f. analyt. Chemie, 1893, p. 252. - Zeitschr. f. Untersuch. Nahr. u. Genussmittel, 1905, t. IX, p. 531. 
nos chifires et ceux d'autres chimistes nous ayant été signalêes de deux côtés à la fois, nous avons procédé à des analyses comparatives et employé pour la première fois une méthode fort appréciée en Hollande : celle de Weibull.

Voici en quoi elle consiste (1):

Méthode de Weibull. - $2 \mathrm{gr} .1 / 2$ de poudre de lait sont mis à bouillir, dans un petit flacon en verre, avee $30 \mathrm{ce}$. d'acide chlorhydrique ( 8 fois normal) et 20 cc. d'eau, en présence d'une pincée de poudre de pierre ponce, pour évityer les soubresauts. L'ébullition doit durer un quart d'heure. On filtre à chaud sur papier mouillé et on lave le résidu resté sur le filtre jusqu'à disparition de toute réaction acide.

Le filtre et son contenu sont desséchés à $100^{\circ} \mathrm{C}$., introduits dans un godet en papier dégraissé et extraits par l'éther dans l'appareil Soxhlet.

L'extrait sec (matière grasse) est desséché à $100^{\circ}$ C. à l'étuve, pendant une demi-heure, et pesé à poids constant.

Cette dernière méthode est assez minutieuse et longue, il faut en convenir. Elle exige beaucoup de pratique et d'attention pour obtenir des résultats exacts et concordants, mais elle présente sur le procédé Gottlieb-Rose l'avantage de n'être pas entachée théoriquement de la cause d'erreur que voici : il arrive, surtout avec des poudres vieilles ou humides, ou celles qui ont subi un commencement de saponification, que le savon formé sous l'addition d'ammoniaque, étant insoluble dans l'éther ordinaire comme dans l'éther de pétrole, entraîne inévitablement une perte de matière grasse échappant au dosage par extraction.

Dans la méthode Weibull, au contraire, l'acide chloryhdrique. employé n'exerce aucune action perturbatrice et la totalité de la matière grasse se retrouve au Soxhlet.

Voici quelques écarts observés par nous-même entre les résultats fournis par les deux méthodes :

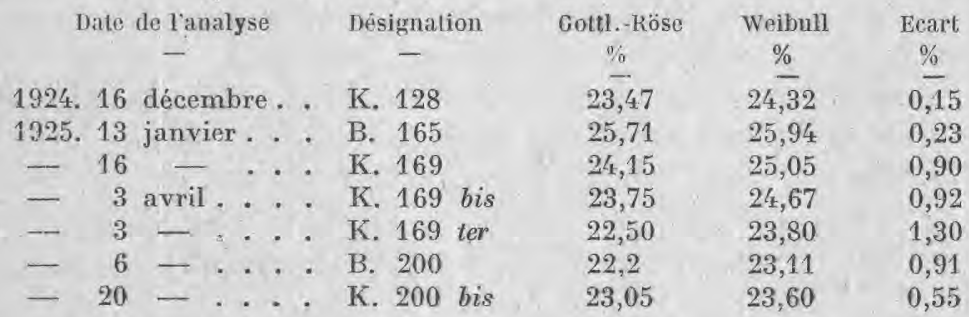

Ces écarts, on le voit, sont loin d'être négligeables dans quelques

(1) Konrng et Mooy, Pharm. Weekblad, 1916, p. 50, 52, 53 (cité par le Codex alimentarius néerlandais, fascicule du Lait, p. 4 et 46 ). 
cas. L'importance de ces divergences, abstraction faite de toute considération théorique, est surtout considérable au point de vue pratique, vu la consommation énorme que l'industrie alimentaire fait de poudres de lait et la taxation de leur valeur marchande au prorata de leur pourcentage en eau et en matière grasse.

En Hollande, on estime que la teneur en graisse d'une poudre de lait entier, rapportée à la matière sèche, doit comporter au moins $23,0 \%$. La teneur en eau ne peut pas dépasser $7 \%$ (Codex alimentarius).

Les petits écarts dans l'expression numérique du pourcentage en matière grasse, rapportěs à des fournitures de stocks très importants - souvent plusieurs tonnes ainsi qu'il est d'usage, - peuvent donner lieu à des déficits énormes.

Pour le chimiste praticien, le dosage exact de la matière grasse d'une poudre de lait est donc une opération de la plus grande importance.

A la suite de l'expérience acquise, nous avons institué des essais comparatifs entre la méthode Weibull ci-dessus décrite et une méthode relativement plus récente, également de source hollandaise, celle de Teichert,

Elle n'est, en somme, qu'une modification de l'ancien procédé butyrométrique de Gerber.

Elle repose sur l'emploi d'un tube butyrométrique spécialement gradué pour l'appréciation du pourcentage en matière grasse des laits en poudre. Dans ce tube spécial, on introduit 2 gr. $1 / 2$ de matière et $10 \mathrm{cc}$. d'acide sulfurique de densité 1,81 , puis 1 ce. d'alcool amylique. On agite, on passe à la centrifuge et on lit le volume de graisse séparée, comme dans le Gerber ordinaire.

Cette méthode présente sur celle de Weibull l'avantage de la simplicité, de la rapidité et du meilleur marché.

Les résultats fournis sont sensiblement concordants, comme le prouvent les chiffres suivants par nous obtenus :

\begin{tabular}{|c|c|c|c|c|c|}
\hline & Date de l'analyse & Désignation & $\begin{array}{c}\text { Weibull } \\
\% \\
-\end{array}$ & $\begin{array}{c}\text { Teichert } \\
\% \\
-\end{array}$ & $\begin{array}{c}\text { Ecart } \\
\% \\
-\end{array}$ \\
\hline 25. & 20 avril. . & k. 201 & 23,6 & 23,75 & 0,15 \\
\hline & $30-$. & k. 202 & 24,08 & 24,2 & 0,12 \\
\hline & 9 mai. & k. 209 & 24,03 & 24,08 & 0,05 \\
\hline
\end{tabular}

Si l'on considère que la méthode primitive de Gerber, comparée à celle de Weibull, accuse des écarts pouvant atteindre jusqu'à $3 \%$ (faits à l'appui mentionnés dans la bibliographie scientifique), on doit reconnaître que le procédé Teichert réalise un heureux perfectionnement.

Les légères différences observées et rapportées ci-dessus tombent 
dans les limites des erreurs possibles, inhérentes à l'imperfection des méthodes analytiques.

En conclusion, ces faits démontrent avec quelle prudence il convient de procéder au choix d'un chimiste que l'on charge d'une analyse dans le domaine si compliqué et si spécial que constitue la science du lait.

\section{LE PROCĖS DE LA MATIÉRE GRASSE DU LAIT} par Ch. PORCHER

- SUite -

Je me suis employé, jusqu'ici, à marquer aussi nettement que possible la distinction qu'il est nécessaire de faire entre le lait individuel et le lait de mélange. Cette distinction, d'ailleurs imposée par tous les documents que nous avons produits dans cette étude, domine l'interprétation des résultats de l'analyse en vue de l'expertise.

Le lait individuel et l'écrémage. - Les variations, en quelque sorte désordonnées du taux de la matière grasse chez un lait individuel, nous mettent dans l'obligation de dire que l'échantillon de comparaison ne peut aroir aucune signification dans le cas de suspicion d'écrémage portant sur un tel lait, même — et la vérité nous dicte le devoir de le dire - si le premier échantillon a été réellement fraudé. Irait-on au plus vite pour prélever le second échantillon, les traites seraient-elles homologues, opérerait-on dans les vingt-quatre heures après la prise du premier, la réponse n'est pas douteuse, a fortiori si les traites sont différentes. Le prélèvement de comparaison n'est ici d'aucune valeur, quelles que soient encore une fois, il est bon de le répéter, les circonstances dans lesquelles il est effectué. C'est même un instrument à deux tranchants. D'un titre élevé en matière grasse, il fera condamner le premier lait, faible, mais qui n'aurait pas été fraudé. Au contraire, d'un titre faible en matière grasse, il contribuera à relever de toutes poursuites un lait antérieur, riche, qui aurait été écrémé. Cette dernière alternative est, en équité, infiniment regrettable, pas plus cependant que la première qui tendrait à faire condamner un innocent; mais je ne lui trouve pas d'autre solution.

Concluons donc de façon ferme, car ici il n'est pas de moyenne mesure, que le prélèvement de comparaison est inopérant lorsqu'il s'agit de juger de l'écrémage d'un lait individuel.

Si maintenant nous quittons le terrain de l'expertise, pour juger à un point de vue plus élevé, physiologique et expérimental, les conséquences que portent en elles les irrégularités du taux de la matière grasse des laits individuels, nous allons voir que celles-ci 